\title{
Stability and Bifurcation Analysis of a Fishery Model with Allee Effects
}

\author{
Makwata Harun ${ }^{1, ~}{ }^{\text {, }}$ Lawi George ${ }^{2}$, Akinyi Colleta ${ }^{2}$, Adu Wasike ${ }^{1}$ \\ ${ }^{1}$ Department of Mathematics and Physical Sciences, Maasai Mara University, Narok, Kenya \\ ${ }^{2}$ Department of Mathematics, Masinde Muliro University of Science and Technology, Kakamega, Kenya
}

Email address:

makwata@mmarau.ac.ke (M. Harun)

${ }^{*}$ Corresponding author

\section{To cite this article:}

Makwata Harun, Lawi George, Akinyi Colleta, Adu Wasike. Stability and Bifurcation Analysis of a Fishery Model with Allee Effects. Mathematical Modelling and Applications. Vol. 4, No. 1, 2019, pp. 1-9. doi: 10.11648/j.mma.20190401.11

Received: March 13, 2019; Accepted: April 26, 2019; Published: May 20, 2019

\begin{abstract}
We study the equilibrium point $\left(n^{*}, E^{*}\right)$ of the fishery model with Allee effect in its population growth dynamics. The Allee effect is considered to be induced by the harvesting of the fish stock. The aggregated model is a set of two differential equations with the fish population and harvesting effort as the dependent variables, with the market price having been taken to evolve faster hence the aggregation from a three dimensional system to a two dimensional system. The analysis of the equilibrium point is performed by looking at three cases in which the threshold population is set at three different values; $T=\frac{n}{4}, T=\frac{n}{2}$ and $T=\frac{3 n}{4}$. Three different equilibrium solutions are obtained: A stable equilibrium, coexistence of three equilibria points with two being saddles and the other stable and the co-existence of three equilibria points with two being stable and a saddle between them. The equilibrium solutions depicts three kinds of fishery: A fishery with fish population maintained at high levels far from extinction but with little economic activity, a fishery with co-existence of an over-exploited and an under-exploited state, which is a dilemma since neither of the state supports sustainable fish resource exploitation, and a fishery that is well managed with fish population being harvested in a sustainable manner thus a balance between commercial harvesting and species existence.
\end{abstract}

Keywords: Allee Effect, Fishing Mortality, Equilibrium Solution

\section{Introduction}

This paper is concerned with the local and bifurcation analysis of the equilibrium point $\left(n^{*}, E^{*}\right)$ of the dynamical system in

$$
\dot{n}=r n\left(\frac{n}{T}-1\right)\left(1-\frac{n}{K}\right)-q n E, \dot{E}=E((A-q n E) q n-c) .
$$

This equilibrium is of interest because its analysis gives the long - term behavior of the system when harvesting is done hence it predicts whether the Fish stock recovers or extinct on harvesting thus guide sustainable harvesting of the fish resource, this is of importance to economists and conservationists. see [11, 13]

There have been great interest in sustainable harvesting of the fish resource in a fishery, see for instance $[1-6,8]$.
Sustainable harvesting has been proposed by using the maximum sustainable yield MSY, which is the maximum proportion that can be removed from the stock over time without causing population decline below optimum level, see for instance [4] This is not necessarily the best management method sine the long run consumption profile does not coincide with that of utility maximization see for instance [8].

A similar equilibrium point is obtained in [1] and [9], but it is the interior point of the system

$$
\begin{aligned}
& E(n)=\frac{r}{q}\left(1-\frac{n}{k}\right) \\
& E(n)=\frac{1}{q n}\left(A-\frac{c}{q n}\right)
\end{aligned}
$$


which yields $E_{1}, E_{2}, E_{3}$, denoting unstable equilibrium, stable equilibrium and the co-existence of three strictly positive equilibria where two are stable, separated by a saddle. The co-existence of three positive equilibria predicts existence of the fishery in either an over-exploited or in an underexploited state which causes a dilemma since the two states cannot co-exist in the same fishery.

\section{Local Stability Analysis}

In model Equation (1), the $n$ nullclines are: $n=0$ and $r\left(1-\frac{n}{k}\right)\left(\frac{n}{T}-1\right)-q E=0$ while the $\mathrm{E}$ nullclines are: $E=0$ and $-c+q n(A-q n E)=0$. The equilibrium points are basically the intersection of $E$ and $n$ nullclines, that is,

$$
\begin{aligned}
& \left(n_{0}, E_{0}\right)=(0,0) \\
& \left(n_{1}, E_{1}\right)=(T, 0) \\
& \left(n_{2}, E_{2}\right)=(k, 0) \\
& \left(n_{3}, E_{3}\right)=\left(n^{*}, E^{*}\right)
\end{aligned}
$$

Our interest is the equilibrium point $\left(n^{*}, E^{*}\right)$ which is the solution of the interior point of the system

$$
\begin{aligned}
& E(n)=\frac{r}{q}\left(\frac{n}{T}-1\right)\left(1-\frac{n}{k}\right) \\
& E(n)=\frac{1}{q n}\left(A-\frac{c}{q n}\right)
\end{aligned}
$$

Whose equilibrium points can be denoted by $E_{1}, E_{2}$, and $\mathrm{E}_{3}$. Equation (1) can be expressed as

$$
\begin{aligned}
& f(n, E):=n r\left(1-\frac{n}{k}\right)\left(\frac{n}{T}-1\right)-q n E, \\
& g(n, E):=-c E+q n E(A-q n E) .
\end{aligned}
$$

The Jacobian matrix is

$$
\begin{aligned}
& J\left(n^{*}, E^{*}\right):=\left(\begin{array}{cc}
f_{n}\left(n^{*}, E^{*}\right) & f_{E}\left(n^{*}, E^{*}\right) \\
g_{n}\left(n^{*}, E^{*}\right) & g_{E}\left(n^{*}, E^{*}\right)
\end{array}\right) \\
& =\left(\begin{array}{cc}
n^{*}\left(\frac{2 r}{T}-\frac{3 r n^{* 2}}{T k}+\frac{2 r}{k}\right)-q E-r & -q n^{*} \\
q E^{*} A-2 q^{2} n^{*} E^{* 2} & -c+q n^{*} A-2 q^{2} n^{* 2} E^{*}
\end{array}\right)
\end{aligned}
$$

To study the nature of the equilibrium point $\left(n^{*}, E^{*}\right)$, we express $c$ as a function of the equilibrium fish population $n^{*}$ by equating the two equations in Equation (4) to obtain

$$
\frac{r}{q}\left(1-\frac{n}{k}\right)\left(\frac{n}{T}-1\right)=\frac{1}{q n}\left(A-\frac{c}{q n}\right)
$$

On expansion and algebraic manipulation, the equation yields

$$
c\left(n^{*}\right)=\frac{r q n^{4}}{T k}-\frac{r q n^{3}}{T}-\frac{r q n^{3}}{k}+r q n^{2}+A q n .
$$

With parameter values set at

$$
r=q=A=1
$$

Equation (7) becomes

$$
c\left(n^{*}\right)=\frac{n^{4}}{T k}-\frac{n^{3}}{T}-\frac{n^{3}}{k}+n^{2}+n
$$

We investigate this Equilibrium point $\left(n^{*}, E^{*}\right)$ by having the threshold population $T$, as a factor of the fish population at a particular time $n$, at three different values, Laboratory results on specific fish species like tuna have shown that if the stock is harvested to a quarter of the stock available at a time, the population recovers to optimum levels in a duration of five years when harvesting is stopped see for instance. We analyze each of this case since harvesting is a continuous process and the results are contained in the propositions and their proofs herein.

\subsection{Case One: $T=\frac{n}{4}$}

With $T=\frac{n}{4}$ Equation (7) becomes

$$
c(n)=\frac{3 r n^{3}}{k}-3 r n^{2}+A n .
$$

Figure 1 shows how c(n) depends on $\mathrm{K}$.

Proposition 2.1

For $T=\frac{n}{4}$ there are three equilibrium points $\left(\mathrm{n}_{\mathrm{i}}^{*}, \mathrm{E}_{\mathrm{i}}^{*}\right)$ with $\mathrm{i}=1,2,3$ satisfying the Equilibrium condition

$$
E^{*}=3 r\left(1-\frac{n^{*}}{k}\right)
$$

Such that $\left(n_{i}{ }^{*}, E_{1}{ }^{*}\right)$ and $\left(n_{2}{ }^{*}, E_{2}{ }^{*}\right)$ being saddle points while $\left(n_{3}{ }^{*}, E_{3}{ }^{*}\right)$ is a stable equilibrium point.

Proof

The Jacobian matrix for this value of $T$ is

$$
J\left(n^{*}, E^{*}\right):=\left(\begin{array}{cc}
\frac{-3 r n}{k} & -q n \\
q E A-2 q^{2} n E^{2} & -2 q^{2} n^{* 2} E^{*}
\end{array}\right) .
$$

The trace is $\operatorname{tr} J(n, E)=-\frac{3 r n}{k}-2 q^{2} n^{* 2} E^{*}<0$.

The determinant $\operatorname{Det} J\left(n^{*}, E^{*}\right)=\frac{3 r}{k} q^{2} n^{* 3} E^{*}+q^{2} n^{*} E^{*}\left(A-2 q n^{*} E^{*}\right)$ which is equal to

$$
\operatorname{Det}\left(n^{*}, E^{*}\right)=q^{2} n^{*} E^{*}\left(\frac{3 r}{k} n^{* 2}-2 q n^{*} E^{*}+A\right)
$$

Using 


$$
E=\frac{3 r}{q}\left(1-\frac{n}{k}\right)
$$

we have

$$
q E^{*}=3 r\left(1-\frac{n^{*}}{k}\right)
$$

which when substituted in the expression for determinant we obtain

$$
\begin{aligned}
& \operatorname{DetJ}\left(n^{*}, E^{*}\right)=q^{2} n^{*} E^{*}\left(\frac{9 r}{k} n^{* 2}-6 n^{*} r+A\right) \\
& =q^{2} n^{*} E^{*}\left(\psi_{1}\right)
\end{aligned}
$$

The derivative of $c(n)=\frac{3 r n^{3}}{K}-3 r n^{2}+n$ with respect to $n$ is $\partial\left(n^{*}\right)=\frac{9 r}{k} n^{* 2}-6 r n^{*}+A$. The solution to this quadratic equation is

$$
n_{1,2}^{*}=\frac{k}{3}\left(1 \pm \sqrt{1-\frac{A}{k r}}\right)
$$

$\partial\left(n^{*}\right)$ is equal to $\psi_{1}$ and $\operatorname{sign} \operatorname{Det} J\left(n^{*}, E^{*}\right)=\operatorname{sign} \psi_{1}$. From the Figure 1 above, we have sign $\partial(n)=\operatorname{sign} \operatorname{Det} J\left(n^{*}, E^{*}\right)>0$ when $n \subseteq\left[n_{2},+\infty\right]$ and $\operatorname{sign} c^{\prime}(n)=\operatorname{sign} \operatorname{Det} J\left(n^{*}, E^{*}\right)<0$ when $n \subseteq\left[0, n_{1}\right] \cup\left[n_{1}, n_{2}\right]$ With $c\left(n_{1}\right)>0$ and $c\left(n_{2}\right)<0$ we have three equilibrium points $\left(n_{1}^{*}, E_{1}^{*}\right),\left(n_{2}^{*}, E_{2}^{*}\right),\left(n_{3}^{*}, E_{3}^{*}\right)$ such that $n_{1}^{*}<n_{1}<n_{2}^{*}<n_{2}<n_{3}^{*}$. For $\left(n_{3}^{*}, E_{3}^{*}\right), n_{2}<n_{3}^{*}, \operatorname{sign} \partial(n)=\operatorname{sign}$ $\operatorname{DetJ}\left(n^{*}, E^{*}\right)>0$ thus stable since $\operatorname{det} J\left(n_{3}^{*}, E_{3}^{*}\right)>0$ and $\operatorname{trJ}\left(n_{3}^{*}, E_{3}^{*}\right)<0$. For $\left(n_{1}^{*}, E_{1}^{*}\right),\left(n_{2}^{*}, E_{2}^{*}\right), n_{1}^{*}<n_{1}<n_{2}^{*} \quad \operatorname{sign}$ $\partial(n)=\operatorname{sign} \operatorname{Det} J\left(n^{*}, E^{*}\right)<0$ thus saddle equilibrium points since $\operatorname{tr} J\left(n^{*}, E^{*}\right)<0$ and $\operatorname{Det} J\left(n^{*}, E^{*}\right)<0$.

\subsection{Case Two: $T=\frac{n}{2}$}

For $T=\frac{n}{2}$ Equation (7) becomes

$$
C(n)=\frac{r n^{3}}{K}-r n^{2}+A n
$$

the graph of Equation (12) shows that as the parameter value $k$ varies the number of Equilibrium point is one for $k<3$ and two more equilibrium points emerges for $k>3$ as seen in Figure 2. In this case, $\partial\left(n^{*}\right)=\frac{3 r}{k} n^{* 2}-2 r n^{*}+A$. The solution $n^{*}$ for $\partial\left(n^{*}\right)=0$ are

$$
n_{1,2}^{*}=\frac{k}{3}\left(1 \pm \sqrt{1-\frac{3 A}{k r}}\right)
$$

Furthermore, The Jacobian matrix with respect to these value of $T$ is

$$
J\left(n^{*}, E^{*}\right)=\left(\begin{array}{cc}
-\frac{r}{k} n^{*} & -q n^{*} \\
q E^{*}\left(A-2 q n^{*} E^{*}\right) & -q^{2} n^{* 2} E^{*}
\end{array}\right)
$$

The trace and the determinant of are; Trace of

$$
J\left(n^{*}, E^{*}\right)=\operatorname{tr}(J)=-\frac{r}{k} n^{*}-q^{2} n^{* 2} E^{*}<0
$$

and

Determinant of

$$
J\left(n^{*}, E^{*}\right)=\operatorname{Det}(J):=q^{2} n^{*} E^{*}\left(\frac{r}{k} n^{* 2}+A-2 q n^{*} E^{*}\right)
$$

respectively. Using

$$
E^{*}=\frac{r}{q}\left(1-\frac{n^{*}}{k}\right)
$$

in $\operatorname{det}(\mathrm{J})$, we obtain

$$
\operatorname{Det}(J)=q^{2} n^{*} E^{*} \psi_{2}\left(n^{*}\right),
$$

where $\psi_{2}\left(n^{*}\right):=\frac{3 r}{k} n^{* 2}-2 r n^{*}+A$. Since $q^{2} n^{*} E^{*}$ is positive, the sign of $\operatorname{Det}(J)$ depends on $\psi_{2}\left(n^{*}\right)$. For $\operatorname{Det}(J), \psi_{2}(n)$ and $\partial(n)$ have the same sign, we have;

$$
\begin{aligned}
& \operatorname{Det}(J)>0 \text { if } n \in\left[0, n_{1}\right] \cup\left[n_{2},+\infty\right], \\
& \operatorname{Det}(J)<0 \text { if } n \in\left(n_{1}, n_{2}\right) .
\end{aligned}
$$

If $r<\frac{3 A}{k}$, then $\partial\left(n^{*}\right)$ is positive and $c\left(n^{*}\right)$ is monotonic increasing with complex roots. If $r>\frac{3 A}{k}$ then there are two real zero's for $\partial\left(n^{*}\right)$. If $r=\frac{3 A}{k}$ the two real zero's coincide. At this point $n_{1,2}^{*}=\frac{k}{3}$, the parameters have further relationship as $c=\frac{k}{9}$ and $E^{*}=\frac{r}{q}\left(1-\frac{n^{*}}{k}\right)=\frac{2 A}{k}$. Further analysis distinguishes two different cases:

Proposition 2.2

For $0<r<\frac{3 A}{k}$ and $k>\frac{c}{A q}, \mathrm{E}_{2}$ is a saddle point and $\left(\mathrm{n}^{*}, \mathrm{E}^{*}\right)$ is a positive stable equilibrium point. 


\section{Proof}

If $0<r<\frac{3 A}{k} 0$ in Equation (13), the sign of $\partial\left(n^{*}\right)$ is positive. Moreover, $\partial\left(n^{*}\right)=\frac{6 r q}{k} n^{*}-2 r q$ implies that $n^{*}=\frac{k}{3}$ is a point of inflection. We have $c(k)=q A k$ but since $c$ is strictly increasing and may take positive or negative values depending on $\mathrm{k}$, we consider $c(k)=q A k-c$ and as $\lim _{n \rightarrow+\infty} c(n)=+\infty$, we conclude that $\mathrm{c}$ vanishes at a unique point $\mathrm{n}^{*}$, thus we obtain a unique equilibrium point $\left(n^{*}, E^{*}\right)$.

If $k<\frac{c}{A q}$, then $c(k)<0$ and $\mathrm{c}$ vanishes at a value $n^{*}>k$, which correspond to the negative effort equilibrium point $\left(E^{*}<0\right)$. In this case, the equilibrium point $E_{2}$ is a stable equilibrium but $\left(n^{*}, E^{*}\right)$ does not present any interest since it is corresponding to unrealistic negative fishing effort, but if $k>\frac{c}{A q}$ then $c(k)>0$ and $\mathrm{c}$ vanishes at a value $n^{*}<k$, with a positive effort equilibrium $\left(E^{*}>0\right)$. In this case $E_{2}$ is a saddle point and $\left(n^{*}, E^{*}\right)$ is the unique positive stable since $\operatorname{det} J\left(n_{3}^{*}, E_{3}^{*}\right)>0$ and $\operatorname{tr} J\left(n_{3}^{*}, E_{3}^{*}\right)<0$.

Proposition 2.3

For $0<\frac{3 A}{k}<r, E_{I}:=\left(n_{i}^{*}, E_{i}^{*}\right)$ for $\mathrm{i}=1,2,3$ are three positive equilibrium points such that we have the following subcases:

If $c\left(n^{*}\right)<0, c\left(n_{1}{ }^{*}\right)<0$, we obtain a unique positive and stable equilibrium point $\left(\mathrm{n}^{*}, \mathrm{E}^{*}\right)$;

If $c\left(n_{1}^{*}\right)>0$ and $c\left(n_{2}^{*}\right)>0$, we obtain a unique positive and stable equilibrium point $\left(\mathrm{n}^{*}, \mathrm{E}^{*}\right)$;

If $c\left(n_{1}^{*}\right)>0$ and $c\left(n_{2}^{*}\right)<0$, we obtain three positive equilibrium points $\left(\mathrm{n}_{\mathrm{i}}^{*}, \mathrm{E}_{\mathrm{i}}^{*}\right)$ for $\mathrm{i}=1,2,3$ whereby $\left(n_{1}^{*}, E_{1}^{*}\right)$ and $\left(n_{3}^{*}, E_{3}^{*}\right)$ are stable while $\left(n_{2}^{*}, E_{2}^{*}\right)$ is a saddle equilibrium point.

$$
0 \leq n_{1}=\frac{k}{3}\left(1-\sqrt{1-\frac{3 A}{r k}}\right)<\frac{k}{3}
$$

and

$$
\frac{k}{3}<n_{2}=\frac{k}{3}\left(1+\sqrt{1-\frac{3 A}{r k}}\right)<k
$$

Recall that $\lim _{n^{*} \rightarrow+\infty} c\left(n^{*}\right)=+\infty$. As $c\left(n_{1}^{*}\right)$ and $c\left(n_{2}^{*}\right)$ can have positive or negative signs, so for subcase 1 , with $c\left(n^{*}\right)<0, c\left(n_{1}^{*}\right)<0$ and $n^{*}>n_{1}, \operatorname{det}(J)>0$ and $\operatorname{tr}(J)<0$ thus a stable equilibrium point $\left(n^{*}, E^{*}\right)$. For Subcase

2, since $c\left(n_{1}^{*}\right)>0$ and $c\left(n_{2}^{*}\right)>0$, with $n^{*}<n_{1}^{*}, \operatorname{det}(J)>0$ and $\operatorname{tr}(J)<0$ thus a stable equilibrium point $\left(n^{*}, E^{*}\right)$. Finally for Subcase 3 , given that $n_{1}^{*}<n_{1}<n_{2}^{*}<n_{2}<n_{3}^{*}$ is satisfied, $\left(n_{1}^{*}, E_{1}^{*}\right)$ and $\left(n_{3}^{*}, E_{3}^{*}\right)$ are stable since $\operatorname{det}(J)>0$ and $\operatorname{tr}(J)<0$ while $\left(n_{2}^{*}, E_{2}^{*}\right)$ is a saddle equilibrium point since $\operatorname{det}(J)<0$ and $\operatorname{tr}(J)<0$.

\subsection{Case Three: $T=\frac{3 n}{4}$}

With $T=\frac{3 n}{4}$ Equation (7) becomes

$$
c(n)=\frac{r n^{3}}{3 k}-\frac{r}{3} n^{2}+A n
$$

Figure 3 shows how $c(n)$ depends on $k$

The derivative of Equation (14) with respect to $n$ is $\partial\left(n^{*}\right)=\frac{r}{k} n^{* 2}-\frac{2}{3} r n^{*}+A$ which vanishes at

$$
n_{1,2}^{*}=\frac{2 k}{3}\left(1 \pm k \sqrt{\frac{1}{9}-\frac{A}{k r}}\right)
$$

The Equation (15) has complex roots not relevant to a realistic fish population.

Proposition 2.4

For $T=\frac{3 n}{4}$ there is one equilibrium point $\left(\mathrm{n}^{*}, \mathrm{E}^{*}\right)$ satisfying the equilibrium condition

$$
E^{*}(n)=\frac{r}{3}\left(1-\frac{n^{*}}{k}\right)
$$

which is stable.

Proof

The Jacobian matrix corresponding to this value of $T$ is

$$
J\left(n^{*}, E^{*}\right):=\left(\begin{array}{cc}
\frac{-r n}{3 k} & -q n \\
q E A-2 q^{2} n E^{2} & -2 q^{2} n^{* 2} E^{*}
\end{array}\right)
$$

The trace is $\operatorname{tr}(n, E)=-\frac{r n}{3 k}-2 q^{2} n^{* 2} E^{*}<0$.

The determinant is

$$
\operatorname{DetJ}\left(n^{*}, E^{*}\right)=\frac{r}{3 k} q^{2} n^{* 3} E^{*}+q^{2} n^{*} E^{*}\left(A-2 q n^{*} E^{*}\right)
$$

which is equal to 


$$
\operatorname{DetJ}\left(n^{*}, E^{*}\right)=q^{2} n^{*} E^{*}\left(\frac{r}{3 k} n^{* 2}-2 q n^{*} E^{*}+A\right)
$$

Using

$$
E=\frac{r}{3 q}\left(1-\frac{n}{k},\right.
$$

in the expression of the determinant, we obtain

$$
\operatorname{DetJ}\left(n^{*}, E^{*}\right)=q^{2} n^{*} E^{*}\left(\frac{r}{k} n^{* 2}-\frac{2}{3} n^{*} r+A\right)=q^{2} n^{*} E^{*}\left(\psi_{3}\right)
$$

The Figure 3 shows that $\partial(n)>0$ and $c(n)$ is monotone increasing thus $c(n)$ vanishes at a unique value $n^{*}<k$. Since the sign of the determinant is the same as the sign of $\psi_{3}$ which is also the sign of $\partial$, the equilibrium point $\left(n^{*}, E^{*}\right)$ is stable since $\operatorname{sign} \partial(n)=\operatorname{sign} \operatorname{Det} J\left(n^{*}, E^{*}\right)<0$.

\section{Bifurcation Analysis}

In this section, we examine the case where $T=\frac{n}{2}$ for $\mathrm{k}=2$, 3 , there is one equilibrium point and when $k=4,5$, two more equilibrium points emerges depicting a bifurcation. The model in Equation (1) when $T$ is replaced with $T=\frac{n}{2}$ it becomes

$$
\begin{aligned}
& \dot{n}=n\left(r\left(1-\frac{n}{k}\right)-q E\right), \\
& \dot{E}=E(-c+q n(A-q n E)),
\end{aligned}
$$

\subsection{Non-dimensionalization}

We non-dimensionalize Equation (18) by making the following transformations

$$
v:=\sqrt{q n}, \mathcal{\varepsilon}:=\frac{\sqrt{q}}{A}, \tau:=\sqrt{q} A t,
$$

and introducing the parameters;

$$
\rho: \frac{r}{A \sqrt{q}}, \gamma:=\frac{c}{A \sqrt{q}}, \kappa:=\sqrt{q} k
$$

By chain rule, the derivative of $n$ is given thus:

$$
\dot{n}=\frac{d n}{d v} \cdot \frac{d v}{d r} \cdot \frac{d r}{d t}=A \frac{d v}{d r}
$$

using (19), (20) and (21) in the first equation of (18) we get

$$
A \frac{d v}{d r}=\frac{v}{\sqrt{q}}\left(\rho A \sqrt{q}\left(1-\frac{v}{k}\right)-\frac{v^{2}}{n^{2}} \cdot \frac{\varepsilon A}{\sqrt{q}}\right)
$$

which upon simplification yields:

$$
\dot{v}=v\left(\rho\left(1-\frac{v}{k}\right)-\varepsilon\right)
$$

where the dot. indicates differentiation with respect to $\tau$. Similarly, the derivative of $E$ is given in dimensionless terms by:

$$
\dot{E}=\frac{d E}{d \varepsilon} \cdot \frac{d \varepsilon}{d \tau} \cdot \frac{d \tau}{d t}=A^{2} \frac{d \varepsilon}{d \tau},
$$

which upon substitution in the second equation of (18) and the use of the dimensionless variables and parameters in (22) and (23), we obtain:

$$
A^{2} \frac{d \varepsilon}{d \tau}=\frac{\varepsilon A}{\sqrt{q}}\left(-\gamma A \sqrt{q}+q \frac{v}{q}\left(A-q \frac{v}{\sqrt{q}} \frac{\varepsilon A}{\sqrt{q}}\right)\right),
$$

that simplifies:

$$
\varepsilon^{\cdot}=\varepsilon(-\gamma+v(1-\varepsilon v))
$$

The model expressed in dimensionless terms becomes:

$$
\begin{aligned}
& \dot{v}=v\left(\rho\left(1-\frac{v}{k}\right)-\varepsilon\right), \\
& \dot{\varepsilon}=\varepsilon(-\gamma+v(1-\varepsilon v)),
\end{aligned}
$$

where the dot denotes differentiation with respect to time $\tau$. Three parameters: $\kappa, \rho, \gamma$ remains. These parameters are interpreted as follows: in case $\rho \ll 1$, and $\gamma \ll 1$, then $A \sqrt{q} \gg$ $r$ and $A \sqrt{q} \gg c$ where we have demand driven overexploitation of the resource. If $\gamma\rangle 1$ and $\rho \gg 1$ then it follows that $A \sqrt{q} \ll\langle r$ and $A \sqrt{q} \ll<c$ which will lead to under-exploitation of the fish resource.

\subsection{Bifurcation}

In this subsection, we compare dimensionless model in Equation (26) to Equation (18), and observe that if we set $r=$ $q=A=1$ in (18), we obtain 20 with $v=n, \varepsilon=E, \rho=r, \gamma=c$ and $\kappa=k$, thus, we use initial parameters $k, c$ and $r$. Bifurcation will show us the long-term dynamic behavior of the aggregated model. We shall show that there is a value of the bifurcation parameter $k=: k_{0}$ where the system in (26) undergoes a saddle-node bifurcation showing the coexistence of two stable equilibria separated by a saddle, whereby the fish population and the fishing effort varies with $k$. This is done by stating and proofing Proposition 3.1 and describing two bifurcation diagrams that show the number and type of stability of points of equilibria as $k$ is varied.

Proposition 3.1

For $\mathrm{n}>2 \mathrm{c}$, there is a value of $\mathrm{k}=: \mathrm{k}_{0}$ where the system in Equation (18) undergoes saddle - node bifurcation as the fish population and the fishing effort dynamics varies with the carrying capacity. Furthermore, for $\mathrm{k}<\mathrm{k}_{0}$, there are only two 
equilibria while when $\mathrm{k}>\mathrm{k}_{0}$, two more equilibria emerge, one stable and the other unstable.

Proof

Using Equations (1) and

$$
\begin{aligned}
& E(n)=\frac{r}{q}\left(1-\frac{n}{k}\right) \\
& E(n)=\frac{1}{q n}\left(A-\frac{c}{q n}\right),
\end{aligned}
$$

and further aggregation, we obtain

$$
\dot{n}=n \varphi(n, k)=: \Phi(n, k)
$$

where

$$
\varphi(n, k):=1-\frac{n}{k}-\frac{1}{n}+\frac{c}{n^{2}}
$$

Clearly, $n=0$ and the curve $\phi(n, k)=0$ gives the equilibrium points. For the stability of the equilibria points $\phi(n, k)=0$, we have

$$
\Phi^{\prime}(\mathrm{n}, \mathrm{k})=\phi(\mathrm{n}, \mathrm{k})+\mathrm{n} \phi^{\prime}(\mathrm{n}, \mathrm{k}),
$$

when $\phi(n, k)=0$, we obtain

$$
\Phi^{\prime}(\mathrm{n}, \mathrm{k})=\mathrm{n} \phi^{\prime}(\mathrm{n}, \mathrm{k}),
$$

where the prime indicates differentiation with respect to $n$. There is stability when $\phi^{\prime}(n, k)<0$ and instability when $\phi^{\prime}(n, k)>0$. Since $\phi^{\prime}(n, k)$ is continuous for $n>0$, we have a change in stability at $\phi^{\prime}(n, k)=0$; that is,

$$
\varphi^{\prime}(n, k)=\frac{-1}{k}-\frac{2 c}{n^{3}}+\frac{1}{n^{2}}=0
$$

or

$$
-n^{3}-2 c k+n k=0
$$

and find that

$$
k=k_{0}:=\frac{n^{3}}{n-2 c},
$$

as the value of $\mathrm{k}$ where a bifurcation occurs.

To be able to indicate the nature of stability in the bifurcation diagram in Figure obtained using (28), consider

$$
n^{\cdot}=n \phi(n, k)=0 \text {. }
$$

The curve $\phi(n, k)=0$ defines equilibrium point $\left(n^{*}, k^{*}\right)$. Clearly

$$
\frac{d \varphi}{d k}=\frac{n^{*}}{k^{2}} \mid\left(n^{*}, k^{*}\right)>0,
$$

thus by the Implicit Function Theorem, there exists

$$
\phi(\mathrm{n}, \mathrm{k}(\mathrm{n}))=0,
$$

$k(n)$ defined in the neighborhood of $\left(n^{*}, k^{*}\right)$ with $k\left(n^{*}\right)=k^{*}$ as smooth as $\phi(n, k)$. Differentiating (30) with respect to $n$, we get

$$
\frac{d \varphi}{d n}+\frac{d \varphi}{d k} \frac{d k}{d n}=0
$$

and hence

$$
\frac{d \varphi}{d n}=-\frac{d \varphi}{d k} \frac{d n}{d n}
$$

from which we can see that

$$
\operatorname{sign}\left(\frac{d \varphi}{d n}\right)=-\operatorname{sign}\left(\frac{d k}{d n}\right)
$$

Hence the nature of stability in the bifurcation diagram in Figure 4 , where the variation of $k$, beyond $k=: k_{0}$ as earlier defined in (28) leads to creation of two more additional equilibrium solutions. This is a bifurcation with the fish population as the variable and the carrying capacity $k$ as the bifurcation parameter.

For the variation of the fishing effort with the carrying capacity as the bifurcation parameter, we obtain where $q=A=1$ in Equation (1). Similarly, we also obtain

$$
n=k(1-E)
$$

from Equation 4.6 such that

$$
\left.k=k_{0}:=\frac{k^{3}(1-E)^{3}}{k(1-E)-2 c},\right)
$$

is the bifurcation value and further aggregation yields

$$
\dot{E}=E \Theta(E, k)=: \Psi(E, k)
$$

where

$$
\Theta(E, k):=-c+k-k E-k^{2} E+2 k^{2} E^{2}-k^{2} E^{3} .
$$

Clearly, $E=0$ and the curve $\Theta(E, k)=0$ gives equilibrium points. For the stability of the equilibrium points $\Theta(E, k)=0$, we have

$$
\Psi^{\prime}(E, k)=\Theta(E, k)+E \Theta^{\prime}(E, k),
$$

and hence

$$
\Psi^{\prime}(\mathrm{E}, \mathrm{k})=\mathrm{E} \Theta^{\prime}(\mathrm{E}, \mathrm{k}),
$$

if evaluated at $\Theta(E, k)=0$. The prime indicates differentiation with respect to $E$. There is stability when $\Theta^{\prime}(E, k)<0$ and instability when $\Theta^{\prime}(E, k)>0$. Since $\Theta^{\prime}(E, k)$ is continuous with $E>0$, there is a change in stability at $\Theta^{\prime}(E, k)=0$. To indicate the nature of stability, consider

$$
E^{\cdot}=E \Theta(E, k)=0 .
$$

The curve $\Theta(E, k)=0$ defines equilibrium points $\left(E^{*}, k^{*}\right)$. 


$$
\frac{d \Theta}{d k}=1-E-2 k E+4 k E^{2}-2 k E^{3},
$$

where it is seen that $\frac{d \Theta}{d k} \mid\left(E^{*}, k^{*}\right)<0$ for $E^{*}>0$. Thus, by the Implicit Function Theorem, there exists

$$
\Theta(\mathrm{E}, \mathrm{k}(\mathrm{E}))=0
$$

$k(E)$ defined in the neighborhood of $\left(E^{*}, k^{*}\right)$ with $k\left(E^{*}\right)=k^{*}$ as smooth as $\Theta(E, k)$. Differentiating Equation (33) with respect to $E$, we obtain

$$
\frac{d \Theta}{d E}+\frac{d \Theta}{d k} \frac{d k}{d E}=0
$$

Thus

$$
\frac{d \Theta}{d E}=-\frac{d \Theta}{d k} \frac{d k}{d E} .
$$

Since $\frac{d \Theta}{d k}<0$, it is evident that

$$
\operatorname{sign}\left(\frac{d \Theta}{d E}\right)=\operatorname{sign}\left(\frac{d k}{d E}\right)
$$

as seen in Figure obtained using (32). The stability changes at $\Theta^{\prime}(E, k)=0$, hence the nature of stability shown. There is existence of only two equilibrium solutions before the bifurcation parameter $k$ passes through the critical value $k=$ : $k_{0}$ defined in (31) where two more equilibrium solutions are generated, one being stable and the other unstable as seen in Figure 5.

\section{Discussion}

We have presented an analysis of the equilibrium point $\left(n^{*}, E^{*}\right)$ of the model in Equation (1) by considering three different values of the threshold population $T$. For $T=\frac{n}{4}$, there is $\left(n_{i}^{*}, E_{i}^{*}\right)$ for $i=1,2,3$ where there are three positive equilibria, two saddles and one stable. For $T=\frac{n}{2}$, there are three cases: Unstable equilibria, stable equilibria and co-existence of three equilibria, two stable separated by a saddle. For $T=\frac{3 n}{4}$, there is only one positive equilibrium which is stable.

The three equilibria with two saddle and a stable predicts a fishery exhibiting management practices to enhance sustainable harvesting. The equilibria $\left(n_{2}^{*}, E_{2}^{*}\right)$ corresponds to harvesting which maintains the stock at values favourable for recovery though unstable since if the management practices are not enforced, the fishery slides to

$\left(n_{1}^{*}, E_{1}^{*}\right)$ where the stock faces a risk of extinction due to huge economic activity. If fishery management practices like moratorium are enforced for the fishery then, the fishery shifts to a stable state of huge fish stock but little economic activity $\left(n_{3}^{*}, E_{3}^{*}\right)$. Co-existence of two stable equilibria separated by a saddle presents a dilemma of coexistence of the fishery in two states: Under-exploited and Over-exploited as discussed in [9].

A positive stable equilibrium predicts a fishery in an under- exploited state in which the stock is huge far from extinction but with minimal economic activity. These results shows that the use of the population growth equation that has a depensation term yields non trivial results in which the equilibria with harvesting now predicts a fishery that can be guided by management practices for sustainable exploitation. Appropriateness of the fishery management practices ensure the state of sustainable harvesting is maintained for the benefit of both economists and conservationists.

\section{Conclusion}

In this work, we have considered a fishery model with Allee effects in the population growth. Analysis of the interior point $\left(n^{*}, E^{*}\right)$ is considering the threshold population at three different values. Results obtained differ significantly with those obtained in the models without Allee effect. The dilemma of the co-existence of the fishery in the overexploited and under - exploited state which both do not support sustainable resource exploitation is resolved by the equilibrium solution that depicts a fishery with sustainable resource exploitation. However, many commercial fish species are highly migratory in search of food and spawning grounds and so is, most harvesting strategy for this species as the fishing vessels moves to zones with high stock density. Therefore, a fishery model with Allee effects in the population growth with the migrations in both the fish stock and the fishing vessels can be considered for further research, since species extinction is a staking reality, see $[7,9,12,14,15]$.

\section{Appendix}

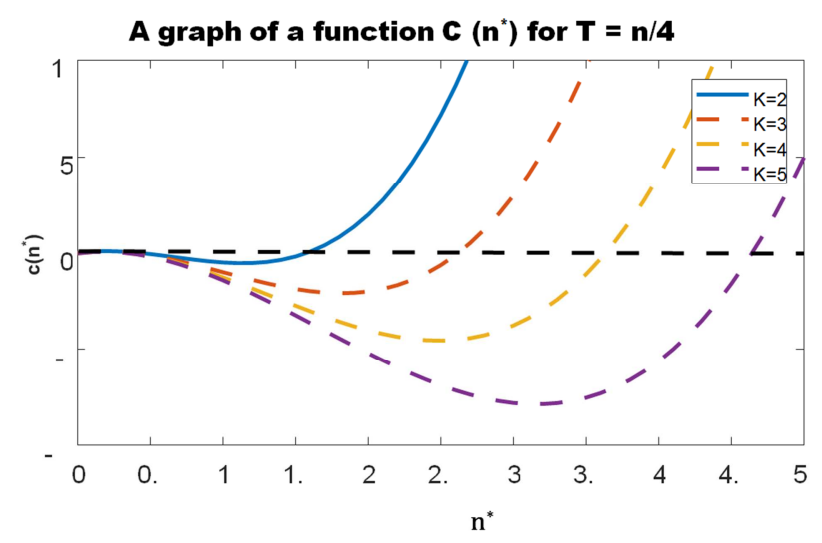

Figure 1. The function $c\left(n^{*}\right)$ plotted for $k=2,3,4$ and 5 . 


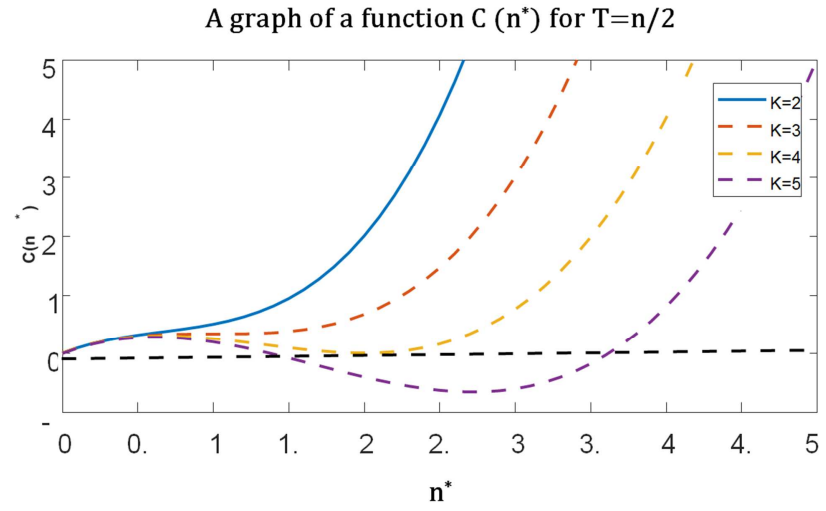

Figure 2. The function $c\left(n^{*}\right)$ plotted for $k=2,3,4$ and 5 .

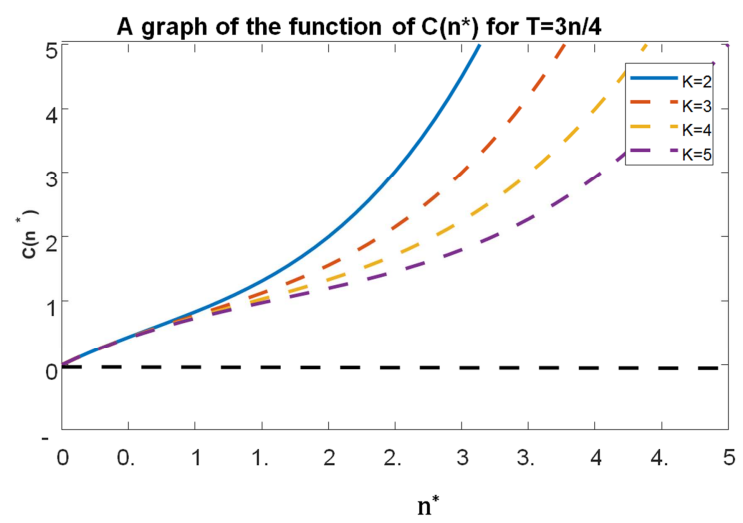

Figure 3. The function $c\left(n^{*}\right)$ plotted for $k=2,3,4$ and 5 .

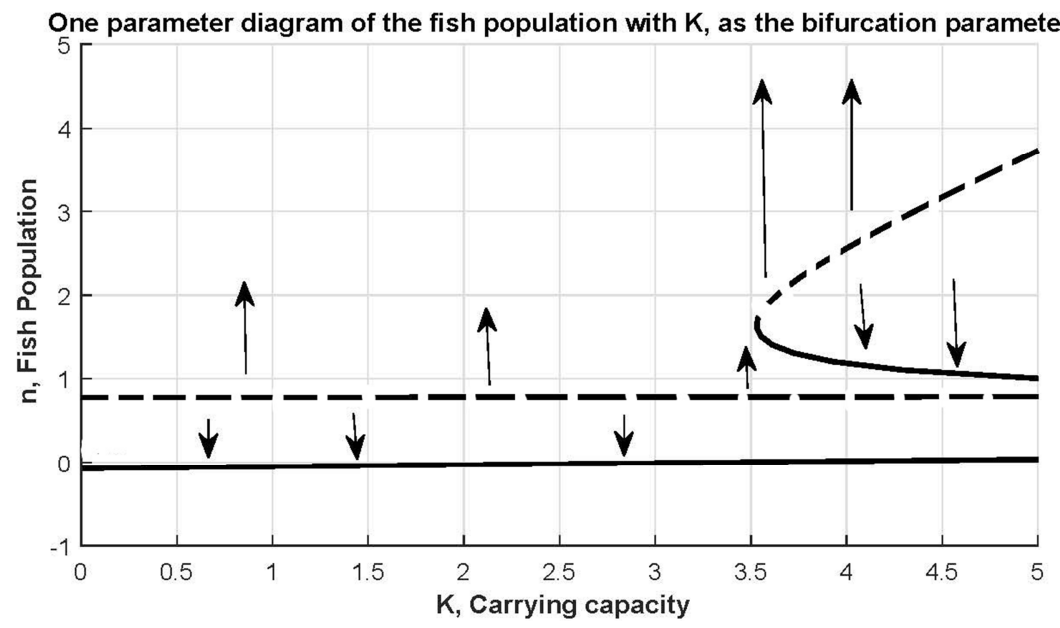

Figure 4. One Parameter bifurcation diagram for fish population with $k$ as parameter.

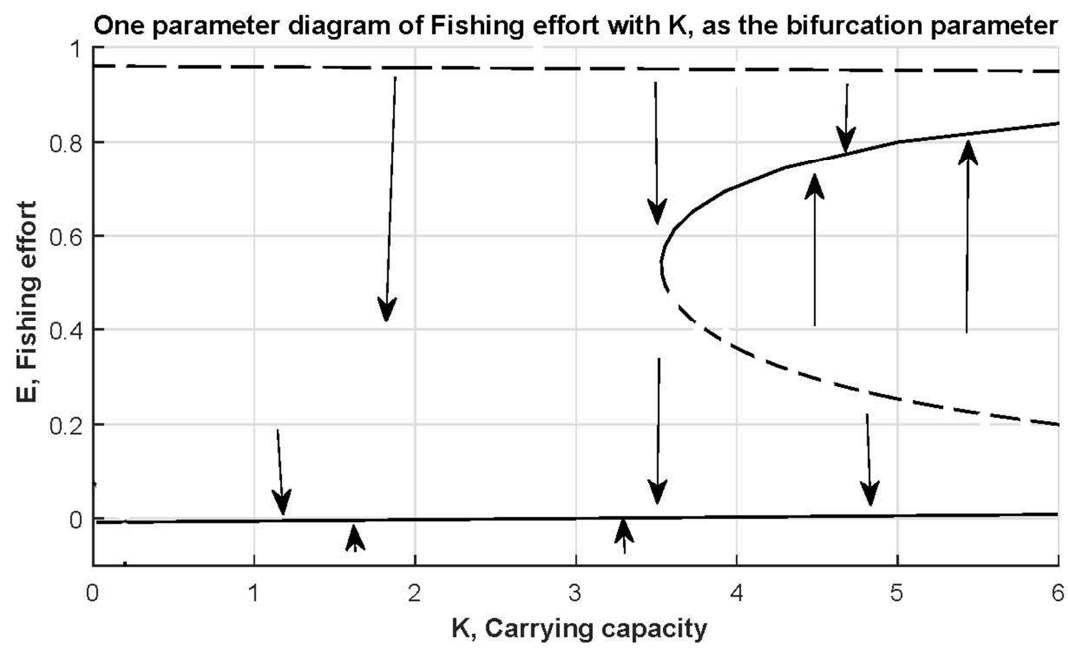

Figure 5. One Parameter bifurcation diagram for Fishing Effort with $k$ as parameter.

\section{References}

[1] Auger P., Mchich R., Nadia R., Kooi B. W. (2009), Effects of market price on the dynamics of a spatial model: Over exploited fishery / Traditional fishery. Ecological complexity.

[2] Auger P. Arnold D. (2009), a model of a fishery with fish stock involving delay equations, Phil, Trans Royal Society A 367.

[3] Barbier E. B., Strand L., Sathirathai S. (2002), Do open access conditions affect the valuation of an externality? Estimating the welfare effects of mangrove-fishery linkages, Env.resour.Econ. 21, 343-367.

[4] Bapan Ghosh et. al (2014), Relationships between exploitation, oscillation, MSY and extinction, Mathematical Biosciences 256, $1-9$. 
[5] Clark C. W. (1990), Mathematical Bioeconomics: The optimal Management of renewable resources, 2nd (Ed), Wiley, New York.

[6] Corrine Wentworth et. al (2011)m Optimum harvesting models for fishery population, Project report, St. Marys' College of Maryland, U. S. A

[7] IUCN 2019, The IUCN red list of threatened species version 20119-1, http://www.iucnredlist.org. Downloaded on 21 march 2019.

[8] Kar T. K. and Matsuda H. (2007), Sustainable Management of a Fishery with a strong Allee effect, Trends In Applied Sciences Research 2(4), 271-283.

[9] Makwata H. et. al (2018), Stability Bifurcation Analysis of a fishery model with nonlinear variation in Market Price, Applied Mathematical SciencesVol. 12, 2018, no. 7 337-350

[10] Mchich R., Auger P. M., Bravo de la parra R., Raissi N.
(2002), Dynamics of a fishery on two fishing zones with fish stock dependent migrations: aggregation and control, Ecological modeling 158(2), 51-62

[11] Murray J. D (2003), Mathematical biology, Springer Verlag Berlin.

[12] Poggiale J. C., Auger P. (1998), Aggregation and Emergence in Systems of Ordinary Differential Equations, Math. comput. Modelling 27(4), 1-21.

[13] Stephens P. A., Sutherland W. J. and Freckleton R. P. (1999), What is Allee effect? Oikos 87(1), 185-190.

[14] Smith V. L (1969), On Models of commercial Fishing, Political economy 77(2), 181-192.

[15] Schaefer M. B (1957. Some considerations of population dynamics and economics in relation to the management of the commercial marine fisheries. J. Fish Res. Board Canada 14, 669-681. 